\title{
Seismic data assimilation with an imperfect model
}

\author{
Miguel Alfonzo ${ }^{1,2}$ (D) . Dean S. Oliver ${ }^{2}$
}

Received: 12 July 2018 / Accepted: 17 June 2019/Published online: 10 July 2019

(C) The Author(s) 2019

\begin{abstract}
Data assimilation methods often assume perfect models and uncorrelated observation error. The assumption of a perfect model is probably always wrong for applications to real systems, and since model error is known to generally induce correlated effective observation errors, then the common assumption of uncorrelated observation errors is probably almost always wrong, too. The standard approach to dealing with correlated observation errors, which simply ignores the correlation, leads to suboptimal assimilation of observations. In this paper, we examine the consequences of model errors on assimilation of seismic data. We show how to recognize the existence of correlated error through model diagnostics modified for large numbers of data, how to estimate the correlation in the error, and how to use a model with correlated errors in a perturbed observation form of an iterative ensemble smoother to improve the quantification of a posteriori uncertainty. The methodologies for each of these aspects have been developed to allow application to problems with very large number of model parameters and large amounts of data with correlated observation error. We applied the methodologies to a small toy problem with linear relationship between data and model parameters, and to synthetic seismic data from the Norne Field model. To provide a controlled investigation in the seismic example, we investigate an application of data assimilation with two sources of model error-errors in seismic resolution and errors in the petro-elastic model. Both types of model errors result in effectively correlated observation errors, which must be accounted for in the data assimilation scheme. Although the data are synthetic, parameters of the seismic resolution and the observation noise are estimated from the actual inverted acoustic impedance data. Using a structured approach, we are able to assimilate approximately 115,000 observations with correlated total observation error efficiently without neglecting correlations. We show that the application of this methodology leads to less overfitting to the observations, and results in an ensemble estimate with smaller spread than the initial ensemble of predictions, but that the final estimate of uncertainty is consistent with the truth.
\end{abstract}

Keywords Model calibration $\cdot$ History matching $\cdot$ Model error $\cdot$ Observation bias $\cdot$ Predictability $\cdot$

Randomized maximum likelihood · Data assimilation · Model improvement · Acoustic impedance $\cdot$ Seismic noise

Norne Field

\section{Introduction}

Data assimilation is the process of modifying uncertain parameters in a model in order to match predictions to data to within a specified tolerance. The term data assimilation is often applied in the weather prediction community to situations in which data are sequentially assimilated as they become available. In this paper, we take the more

Miguel Alfonzo

Miguel.Alfonzo@norceresearch.no

1 University of Bergen, Bergen, Norway

2 Norwegian Research Centre (NORCE), Bergen, Norway general approach in which all data might be assimilated simultaneously. In this case, data assimilation for reservoir models becomes synonymous with history matching [44, 45]. Data assimilation also generally implies a Bayesian approach to uncertainty estimation [20], which we take as a key component. In order to use a Bayesian approach, it is necessary to characterize the prior uncertainty in model parameters, the accuracy of the observations and limitations in the model so that one is able to weight the observations appropriately. If the magnitude of the data mismatch after model calibration is larger than expected based on measurement error, then one might conclude that the difference is at least partially due to deficiencies in the model: missing physical processes, missing parameters, or errors in the forward model. 
Model error is an inherent problem in all real-world applications of data assimilation. In this paper, our focus is on a methodology for treatment of model error whose origin is unknown or of a type for which removal of the source of error is impractical. In the examples, the actual model error is of two types. The first is often termed "error of representation' [28, 52]. This error is often a result of the neglect of small-scale processes and heterogeneity. To mimic representation error, we examine situations in which the data are obtained from a system with a finer resolution than the predictive model. The second source of error in the examples is an error in parameters of the forward simulator.

In applications of seismic history matching, model error may occur because of errors in the petro-elastic model (PEM), inappropriate filtering, missing parameters, or many other factors [58]. Furthermore, there exists differences in resolution (frequency content) between seismic data and seismic predictions made from the PEM and reservoir simulation models. On the one hand, seismic data shows a higher lateral resolution than that of simulation models. Therefore, for better match with seismic observations, it is common practice to downscale simulation results (e.g., pressures and saturations) to the resolution of the finer reservoir model prior to estimation of impedances $[50,55]$. On the other hand, different approaches have been proposed to address the issue of the limited vertical resolution of seismic data [16]. These include the use of stochastic seismic inversion workflows to improve the vertical resolution of seismic [24, 32, 36], or Bayesian downscaling of inverted impedance data [4]. Furthermore, due to discontinuities in the simulation model (gridding, faults, etc.), seismic predictions may contain high frequency features that are outside of the frequency spectrum of the actual seismic data [2]. In this paper, we perform the comparison between seismic observations and predictions at the seismic scale, so no downscaling of simulations results or seismic data is needed. Instead, seismic predictions are filtered (smoothed) within the seismic frequency bandwidth [50].

In history matching of production data and in numerical weather prediction, model error is often either ignored or treated in an ad hoc manner by simply inflating the observation error covariance beyond the actual measurement error level $[7,11]$. While this approach can reduce the tendency to underestimate uncertainty, it also tends to exclude smallscale information in the data $[49,57]$. The effect of model error is more difficult to ignore when assimilating seismic data, partially because the amount of data is often much larger than when only assimilating production data, and partially because the information provided by the seismic appears to be complimentary to the information provided by the production data [56]. When the amount of data is large, as it is for seismic, even small amounts of model error can have large impact on the results of data assimilation [13, 39]. We note that errors in production data tend to be correlated in time [22], while errors in seismic data tend to be spatially correlated [48]. Since all data in history matching to production and seismic data are assimilated simultaneously, the type of correlation (spatial or temporal) is irrelevant to the process.

There are at least three unfortunate consequences of ignoring model error in data assimilation: (1) parameters of the model are adjusted to incorrect and often implausible values, (2) the uncertainty in predictions tend to be underestimated, and (3) the forecasts are biased [5, 15, 64]. Although the importance of recognizing model error and its effect on correlation of observation errors is widely recognized [54, 60, 61], methods for treating model error and the subsequent autocorrelation in residual data errors are uncommon in practice. Accounting correctly for the effects of autocorrelation in the observation error model has, however, been shown to significantly improve calibrated parameter values and uncertainty estimates [23].

There are many ways to identify the presence of significant model error, but one standard method for diagnosing a model is through comparison of the model predictions with actual observations [31, 63]. If the model parameters that we generate do not in fact reproduce the data within the expected tolerance, then we must conclude that either the data assimilation has been done poorly, or that the assumptions that were made about the magnitude of the measurement error were wrong, or that the forward model might be incapable of reproducing important processes. Unfortunately, most methods for evaluating the expected value of a model diagnostic based on the magnitude of the residuals require the inverse of the observation error covariance matrix [38], which is difficult when the errors are correlated and the number of data is large.

When a model has been judged to be deficient, the first course of action would be to attempt improvement by adding model parameters, reassessing the prior uncertainty, refining the model discretization, or by including physical processes that were previously neglected. Typically, however, there is a limit to improvements that can be made to the model and when that limit has been reached, the model may still be noticeably imperfect. The only available recourse at this point is to reduce the expectation of the quality of the match of the model to the data. Modification of the estimate of the observation error covariance matrix, $\mathbf{C}_{D}$, can be used to modify the relative weighting of various types of data and prevent overly large changes in the model parameters in an attempt to make predictions from a imperfect model match data [29, 37].

We previously demonstrated the benefits of a workflow that includes model criticism and iterative model improvement on a small flow problem with model error due to 
onset of turbulence and errors in measurement [43]. The methodology was shown to result in better quantification of uncertainty and in more robust forecasts. Because the test problem was small in those examples, the ability to apply the methodology to large reservoir problems was not obvious. In this paper, we present new methods for treating several aspects of the problem of data assimilation with imperfect models that are significantly more difficult when the number of model parameters and the number of data are large, as is the case when assimilating seismic data: recognition of the need for model improvement through evaluation of a model diagnostic for the posterior ensemble, the iterative improvement of an estimate of the total observation error covariance, the generation of samples of correlated observation errors, and a methodology for computation of the gain matrix when observation errors are correlated.

\section{Methodology}

In this section, we summarize key elements of a methodology for dealing with correlated observation errors in large data assimilation problems for which formation of the observation error covariance matrix may be problematic and for which sampling of noise from the observation noise distribution will be difficult. We also describe a methodology for checking the posteriori ensemble by comparing the magnitude of data mismatch with the expected magnitude. Finally, we develop a method for updating the estimate of the total observation error that accounts for the presence of model error.

\subsection{Assimilation of data with non-diagonal $C_{D}$}

The most straightforward approach to the use of correlated observation error (non-diagonal observation error covariance matrix $\mathbf{C}_{D}$ ) in ensemble-based data assimilation is to simply generate perturbed observations with correlated errors, then use a low-rank representation of the observation error covariance matrix as described by Evensen [20, sec. 14.3]. Instead of forming the non-diagonal matrix $\mathbf{C}_{D}$, we generate an ensemble of independent realizations of correlated observation error $\mathbf{E}$ from the distribution $N\left(\mathbf{e} \mid 0, \mathbf{C}_{D}\right)$, then use the sample covariance matrix $\mathbf{E E}^{\mathrm{T}} /\left(N_{e}-1\right)$ to represent the data error covariance matrix $\mathbf{C}_{D}$, where $N_{e}$ is the number of ensemble members, or samples. An equation for updating the ensemble of parameters $\boldsymbol{\Theta}^{\mathrm{pr}}$ for consistency with observations $\mathbf{d}_{\mathrm{obs}}$ can be obtained easily in the linear case.

$$
\begin{aligned}
\boldsymbol{\Theta}^{\text {post }}= & \boldsymbol{\Theta}^{\mathrm{pr}}+\Delta \boldsymbol{\Theta}^{\mathrm{pr}}(\Delta \mathbf{D})^{\mathrm{T}}\left[\Delta \mathbf{D}(\Delta \mathbf{D})^{\mathrm{T}}+\mathbf{E} \mathbf{E}^{\mathrm{T}}\right]^{-1}\left(\mathbf{d}_{\mathrm{obs}}-(\mathbf{D}+\mathbf{E})\right) \\
\approx & \boldsymbol{\Theta}^{\mathrm{pr}}+\Delta \boldsymbol{\Theta}^{\mathrm{pr}}(\Delta \mathbf{D})^{\mathrm{T}} \mathbf{U}_{0} \mathbf{W}_{0}^{-\mathrm{T}}\left(\mathbf{I}+\mathbf{W}_{0}^{-1} \mathbf{U}_{0}^{\mathrm{T}} \mathbf{E E}^{\mathrm{T}} \mathbf{U}_{0} \mathbf{W}_{0}^{-\mathrm{T}}\right)^{-1} \mathbf{W}_{0}^{-1} \mathbf{U}_{0}^{\mathrm{T}} \\
& \quad \times\left(\mathbf{d}_{\mathrm{obs}}-(\mathbf{D}+\mathbf{E})\right)
\end{aligned}
$$

where $\Theta^{\text {post }}$ is the ensemble of model parameters conditioned to observations, $\Delta \Theta^{\mathrm{pr}}$ is the matrix of deviations of $\boldsymbol{\Theta}^{\mathrm{pr}}$ from the mean of the ensemble, $\mathbf{D}$ is the matrix whose columns are realizations of predicted data, and $\Delta \mathbf{D}$ is the matrix of deviations of $\mathbf{D}$ from the mean of the ensemble. We have used a truncated singular value decomposition (SVD)

$$
\Delta \mathbf{D} \approx \mathbf{U}_{0} \mathbf{W}_{0} \mathbf{V}_{0}^{\mathrm{T}}
$$

in the second line of Eq. 1. Although the number of data $\left(N_{d}\right)$ will be quite large in practical cases, it is only necessary to compute the SVD of a matrix of dimension $N_{d} \times N_{e}$. The effort required for computation is $O\left(N_{d} N_{e}^{2}\right)$ [59], which is only linear in the number of data.

Algorithm 1 summarizes the steps for data assimilation with correlated observation error. To make the assimilation feasible for large models and for large amounts of data, we apply localization of the Kalman gain matrix. Although there will be efficiency gain from using local analysis
[8], in Algorithm 1 we simply assume that updates of model parameters are computed row-by-row so that it is not necessary to form the entire Kalman gain matrix [17]. Also, in any practical application, one would replace the Algorithm 1 with an iterative ensemble smoother (e.g., $[6,18])$ and one would apply scaling to the data before computation of the truncated SVD. For clarity of exposition, none of these important steps are shown here.

\subsection{Evaluation of a model diagnostic for criticism of the posterior ensemble}

A very standard way of evaluating history matched models is to compare predictions from the ensemble of calibrated models with observations. In order to determine if the magnitude of data residuals after calibration is consistent with model assumptions, we compute a particular model diagnostic

$$
\tilde{S}_{d}^{\text {obs }}=\left(\mathbf{h}\left(\boldsymbol{\theta}^{\text {post }}\right)-\mathbf{d}^{\text {obs }}\right)^{\mathrm{T}} \mathbf{C}_{D}^{-1}\left(\mathbf{h}\left(\boldsymbol{\theta}^{\text {post }}\right)-\mathbf{d}^{\text {obs }}\right)
$$




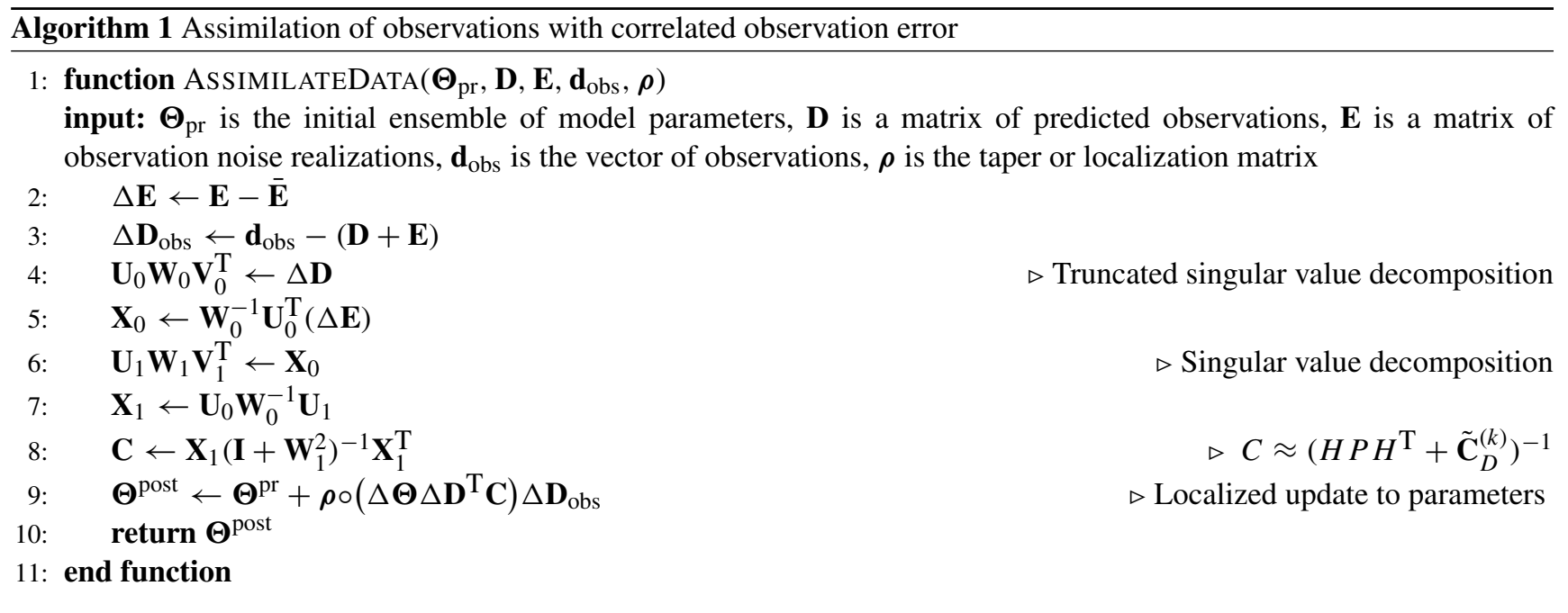

where $\mathbf{h}$ is the observation operator that defines the relationship between model parameters and data predictions, and $\boldsymbol{\theta}^{\text {post }}$ is a sample of model parameters from the posteriori distribution, after conditioning to data. Note that $\tilde{S}_{d}^{\text {obs }}$ evaluates the mismatch between calibrated realizations and the actual observations. If the model is correctly specified and the minimization is performed well, then the expected magnitude of $\tilde{S}_{d}^{\text {obs }}$ is $N_{d}$.

When $\mathbf{C}_{D}$ is diagonal or when the number of data is small, computation of the model diagnostic $\tilde{S}_{d}^{\text {obs }}$ is trivial. When the number of data is large and when the observation errors are correlated, however, computation is not so straightforward. One possible approach for observation errors with stationary covariance is to take advantage of the matrix structure to compute the inverse efficiently [40, 42]. In our methodology, however, the observation error covariance matrix itself is never computed. Instead, it is approximated from an ensemble of residuals. Our metric is then similar to the Mahalanobis distance, except for the fact that the sample covariance is not full rank, so it is not invertible.

In those cases where an estimate of the magnitude of $\tilde{S}_{d}^{\text {obs }}$ is needed, but the representation of $\mathbf{C}_{D}$ is not full rank, there are several options for computing an approximation of $\mathbf{C}_{D}^{-1}$. The most obvious is to simply use the pseudoinverse of $\mathbf{E E}^{\mathrm{T}} /\left(N_{e}-1\right)$ but if this approach is taken, the expected value of $\tilde{S}_{d}^{\text {obs }}$ is no longer $N_{d}$. Engel et al. [19] provide a recent survey of methods for estimating the precision matrix (the inverse of the covariance matrix) for the case in which the number of parameters is large and the number of samples is small. We have chosen to shrink the low-rank sample covariance estimate towards the diagonal matrix whose diagonal elements are all equal to the average of the diagonal elements of the sample covariance matrix $[34,53]$. If the variance is thought to be nonstationary, then it might be appropriate to use a different target, e.g., Target D: "diagonal, unequal variance" [53].
Let the shrinkage-based estimate of the covariance matrix for vector $x$ be

$\hat{\mathbf{\Sigma}}=\delta(v \mathbf{I})+(1-\delta)\left(\frac{1}{N_{e}-1} \mathbf{X X}^{\mathrm{T}}\right)$

where $\nu \mathbf{I}$ is the target covariance matrix, $\mathbf{X}$ is the $N_{d} \times N_{e}$ matrix whose columns are mean-removed random samples of the vector $x, \mathbf{X X}^{\mathrm{T}} /\left(N_{e}-1\right)$ is the sample covariance matrix, and $\delta$ is the shrinkage parameter. Since our choice of the target matrix is diagonal, the formula for the inverse can be simplified using the Sherman-Morrison-Woodbury formula.

$$
\begin{aligned}
\hat{\mathbf{\Sigma}}^{-1} & =\frac{1}{\delta v} \mathbf{I}-\frac{1}{\delta^{2} v^{2}}\left(\frac{1-\delta}{N_{e}-1}\right) \mathbf{X}\left(\mathbf{I}+\frac{1}{\delta v}\left(\frac{1-\delta}{N_{e}-1}\right) \mathbf{X}^{\mathrm{T}} \mathbf{X}\right)^{-1} \mathbf{X}^{\mathrm{T}} \\
& =\frac{1}{\delta v} \mathbf{I}-\frac{1}{\delta v} \mathbf{X}\left(\delta v\left(\frac{N_{e}-1}{1-\delta}\right) \mathbf{I}+\mathbf{X}^{\mathrm{T}} \mathbf{X}\right)^{-1} \mathbf{X}^{\mathrm{T}} .
\end{aligned}
$$

Schäfer and Strimmer [53] discuss a method for estimating the optimal shrinkage, but since it involves computation of the sample covariance, we instead use the simpler estimate of Leung and Chan [35]

$\delta^{*}=\frac{2}{N_{e}+2}$.

For this particular shrinkage target, an estimate of the Mahalanobis distance can be computed efficiently,

$$
\begin{aligned}
D_{M}^{2}(\mathbf{x})= & (\mathbf{x}-\hat{\boldsymbol{\mu}})^{\mathrm{T}} \hat{\boldsymbol{\Sigma}}^{-1}(\mathbf{x}-\hat{\boldsymbol{\mu}}) \\
= & \frac{1}{\delta v}(\mathbf{x}-\hat{\boldsymbol{\mu}})^{\mathrm{T}}(\mathbf{x}-\hat{\boldsymbol{\mu}})-\frac{1}{\delta v}\left(\mathbf{L}^{-1} \mathbf{X}^{\mathrm{T}}(\mathbf{x}-\hat{\boldsymbol{\mu}})\right)^{\mathrm{T}} \\
& \left(\mathbf{L}^{-1} \mathbf{X}^{\mathrm{T}}(\mathbf{x}-\hat{\boldsymbol{\mu}})\right)
\end{aligned}
$$

where we have performed a Cholesky factorization of $\left(\delta v\left(\frac{N_{e}-1}{1-\delta}\right) \mathbf{I}+\mathbf{X}^{\mathrm{T}} \mathbf{X}\right)=\mathbf{L L}^{\mathrm{T}}$. Note that the matrix that requires factorization (or inversion) is generally quite small 
$\left(N_{e} \times N_{e}\right)$ even for very large data assimilation problems, so computation is very fast.

Although the use of shrinkage provides an improved estimate of both $\mathbf{C}_{D}$ and $\mathbf{C}_{D}^{-1}$, the magnitude of $D_{M}^{2}(\mathbf{x})$ obtained using Eq. 4 may still be far from the value that would be obtained using the true value of $\mathbf{C}_{D}^{-1}$, so direct comparison of $D_{M}^{2}(\mathbf{x})$ with the number of data is not possible. As a final step, we compute an empirical probability distribution for values of $D_{M}^{2}(\mathbf{x})$ based on samples from the true distribution. Denoting the ensemble of residuals after data assimilation with perturbed observations by $\mathbf{r}$ (that is the $i$ th column of $\mathbf{r}$ is the mismatch $\left.\mathbf{r}_{i}=\mathbf{h}\left(\boldsymbol{\theta}_{i}^{\text {post }}\right)-\mathbf{d}^{\text {obs }}\right)$ and the ensemble of observation perturbations by $\mathbf{E}$, we apply the function Computediagnostic(E, r) from Algorithm 2 to obtain two distributions of squared Mahalanobis distances. One distribution corresponds to realizations of data residuals (the columns of $\mathbf{r}$ ) and the second distribution corresponds to independent samples of the observation error (derived from the columns of E). $p$ in Algorithm 2 denotes the probability that the ensemble of observation perturbations $\mathbf{E}$ is drawn from the same distribution as the ensemble of data residuals r. Larger values of $p$ therefore suggest that the distance between the two distributions is smaller, and hence that $\mathbf{E}$ is more likely to be drawn from the same distribution as r. Our metric is then based on the modified Z-score computed for the distance between the medians of the two distributions [30]. Other nonparametric tests, such as the two-sample Kolmogorov-Smirnov test, might be more appropriate. The steps are summarized in Algorithm 2.

\subsection{Generation of perturbed observations}

In the perturbed observation forms of the iterative ensemble smoothers with model improvement, it is necessary to generate ensembles of observation perturbations multiple times. When the observation errors are independent, the cost is small, but when the errors are correlated then the cost of generating the perturbations for large data sets can be significant. In our applications, we use two different methods depending on the representation of the estimate of the observation error covariance matrix.

In most cases, the initial estimate of observation error covariance will be obtained using an assumption of stationarity so that standard methods for generating unconditional realizations of gaussian random fields can be employed. Because the size of the data may be large, we use the method of circulant embedding of the covariance matrix

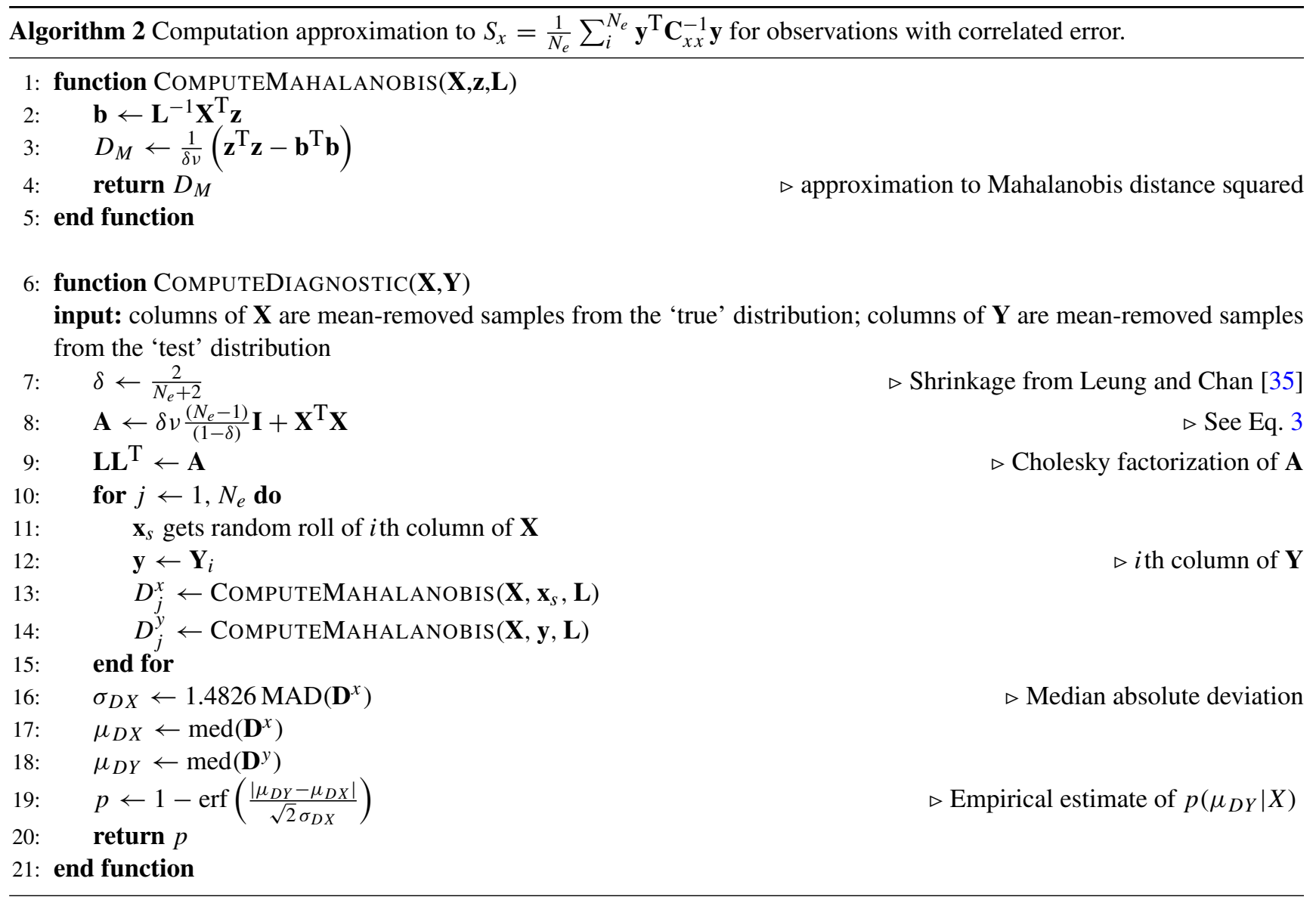


[14] to generate observation perturbations. The key to the efficiency of the algorithm is that the covariance matrix is symmetric block-circulant with circulant blocks and is completely specified by its first block row [33].

An alternative method for generating samples of total observation error is required after updating the estimate of
$\mathbf{C}_{D}$. Following the methodology of Oliver and Alfonzo [43], we compute the updated estimate of the total covariance from the ensemble of data mismatch between model predictions and actual observations after data assimilation with perturbed observations. Thus, if

$\boldsymbol{\theta}_{i}^{\text {post }}=\underset{\theta}{\operatorname{argmin}}\left[\left(\boldsymbol{\theta}-\boldsymbol{\theta}_{i}^{\mathrm{pr}}\right)^{\mathrm{T}} \mathbf{C}_{\theta}^{-1}\left(\boldsymbol{\theta}-\boldsymbol{\theta}_{i}^{\mathrm{pr}}\right)+\left(\mathbf{h}(\boldsymbol{\theta})+\boldsymbol{\epsilon}_{i}-\mathbf{d}^{\mathrm{obs}}\right)^{\mathrm{T}} \mathbf{C}_{D}^{-1}\left(\mathbf{h}(\boldsymbol{\theta})+\boldsymbol{\epsilon}_{i}-\mathbf{d}^{\mathrm{obs}}\right)\right]$

where $\boldsymbol{\epsilon}_{i}$ is a perturbation to the predicted observations and $\boldsymbol{\epsilon}_{i} \sim N\left[0, \mathbf{C}_{D}\right]$ and if the residual for updated ensemble member $\boldsymbol{\theta}_{i}^{\text {post }}$ is defined to be (see Appendix)

$\mathbf{r}_{i}=\mathbf{h}\left(\boldsymbol{\theta}_{i}^{\text {post }}\right)-\mathbf{d}^{\mathrm{obs}}$,

then the maximum likelihood estimate of the total observation error covariance is

$\hat{\mathbf{C}}_{D}=\frac{1}{N_{e}} \mathbf{r r}^{\mathrm{T}}$

where $\mathbf{r}=\left(\mathbf{r}_{1}, \mathbf{r}_{2}, \ldots, \mathbf{r}_{N_{e}}\right)$. In all practical cases involving seismic data, $N_{e} \ll N_{d}$ so the maximum likelihood estimate of $\mathbf{C}_{D}$ will be rank deficient. If, however, all we need are samples of observation error from $\hat{\mathbf{C}}_{D}$ for the perturbed observation data assimilation, then it is not necessary to estimate a full rank $\hat{\mathbf{C}}_{D}$ to generate new realizations, as the $\mathbf{r}_{i}$ are themselves samples from $\hat{\mathbf{C}}_{D}$.

If only a single ensemble of realizations from $N\left[\boldsymbol{\epsilon} \mid 0, \hat{\mathbf{C}}_{D}\right]$ is required, it would be possible to use the columns of $\mathbf{r}$. In some methods, such as the perturbed observation form of multiple data assimilation (MDA), however, it is necessary to generate independent perturbations at each iteration [18]. To generate an additional ensemble of samples, we apply a transformation that generates a new ensemble of observation perturbations from the original ensemble by circularly shifting and recombining perturbations. The circular shift simply moves elements from one position on the grid to another. The random recombination recombines the realizations without affecting the covariance.

The method that uses shifting to generate new realizations is very fast and does not require estimation or fitting of covariance models. It is limited, however, to rectangular regular grids. Also, the shift introduces a discontinuity in the perturbation that may in some cases be significant. It is not noticeable in our applications because it is followed by the random recombination step from a QR factorization of a random matrix. The methodology is summarized in Algorithm 3.

\subsection{Model improvement workflow}

The functionalities described in Algorithms 1-3 are parts of a structured data assimilation methodology that provides for both model criticism and model improvement. A fairly complete, but necessarily simplified workflow is shown in Algorithm 4. In summary, this workflow comprises data assimilation with correlated observation errors (line 6, Algorithm 1), model diagnostic for the posterior ensemble (line 8, Algorithm 2), the iterative estimation of the total observation error from the data residuals, and the generation of samples of correlated observation errors (lines 7 and 9, Algorithm 3). The model improvement cycles stop when the model diagnostics suggests that the model is adequate (the value of the probability $p$ is larger than the threshold of 0.05 , or any other defined threshold), or the maximum number of user-defined iterations $\left(k_{\max }\right)$ is reached.

In practice, each of the steps in the workflow is more complex than described in this paper. For example, it is common to perform a first check of the adequacy of the initial ensemble against the actual observations. One possible test is to evaluate the probability that the observations are drawn from the same distribution as the ensemble of model predictions (function CHECKMODEL in Algorithm 4, not shown in this paper). If this test suggests that the ensemble of model predictions is inconsistent with the observations (low probability values, $p^{\text {init}}$ ), one should attempt to improve the model prior to data assimilation (lines 1 to 3 in Algorithm 4). Possible avenues for model improvement may include the addition of new model parameters, modification of the prior distribution of model parameters, or modification of the distribution of observation errors. Furthermore, for any practical subsurface data assimilation problem, it is necessary to apply an iterative data assimilation method to deal with nonlinearity instead of the ensemble smoother method used for illustration in Algorithm 1. In our seismic test case, we have applied ES-MDA [18]. In that case, after each cycle of data assimilation, the data perturbations must be randomized and rescaled by an inflation factor $\alpha$. If, instead an iterative ensemble smoother (e.g., [6]) is used for the data assimilation, then a scalar inflation would be applied at each iteration, but resampling the data noise would not be necessary. Also, in Step 9 of Algorithm 1, we show gain localization for the entire gain matrix. For large data assimilation problems, it is impractical to form the entire 

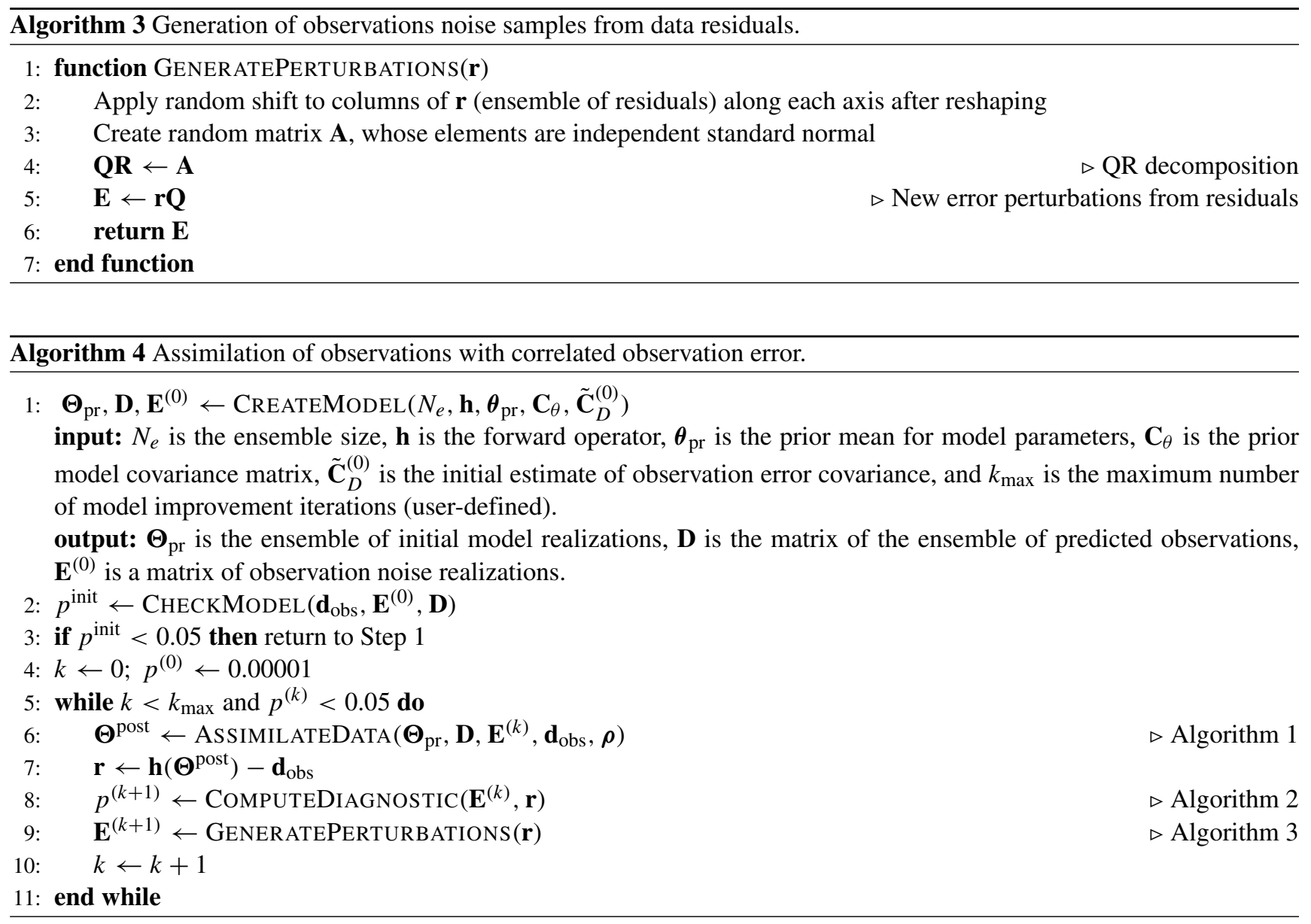

gain matrix. In the seismic test problem, the gain matrix is formed one row at a time (as in [17]) (or the analysis can be done using local analysis, updating model parameters column-by-column [8]). Local analysis with observation tapering is used for the linear 1D example.

\section{Example applications}

We illustrate the methodology with two numerical examples. In the first example, the observations are linear and the prior distribution is Gaussian, so the posterior distribution will also be Gaussian. The difficulty is that the observation operator (i.e., the true sensitivity of the data to the model parameters) is different from the observation operator that is assumed for the data assimilation. The problem is small enough that the results can be examined analytically and the true total covariance can be computed. The second example is based on synthetic seismic data for the Norne Field case. Here, the number of data is large so that it becomes impractical to compute the inverse of the covariance matrix when the observation errors are correlated. Model error affects the synthetic seismic through an error in the petro-elastic model and through inaccuracy of the observation operators.

\subsection{Linear 1D test case}

The synthetic truth for this test problem is a realization of a correlated gaussian random vector of length 150 with prior mean 0 and exponential covariance

$\operatorname{cov}\left(x, x^{\prime}\right)=4 \exp \left(-\frac{3\left|x-x^{\prime}\right|}{25}\right)$.

Observations of the truth are made in every second lattice point with independent measurement errors characterized by variance 0.04 .

To mimic the effect of error in the forward model, we incorrectly assume that the observations were obtained from a smoothed model (Hann filter with width 31). Thus the approximate observation operator is

$\tilde{h}\left(x_{n}\right)=\sum_{i=-15}^{15} w_{i} x_{n-i} \quad$ for $\quad w_{i}=(1+\cos (2 \pi i / 30)) / 30$

while the true observation operator is $h\left(x_{n}\right)=x_{n}$. This is roughly equivalent to using a coarse model for data assimilation. Figure 1a shows the true property field and the noisy observations from that field. Figure $1 b$ shows the true observation operator (a delta function) and the approximate 
Fig. 1 The linear 1D example with model error. a The true property field and the noisy observations. b True and approximate observation operators for $n=75$

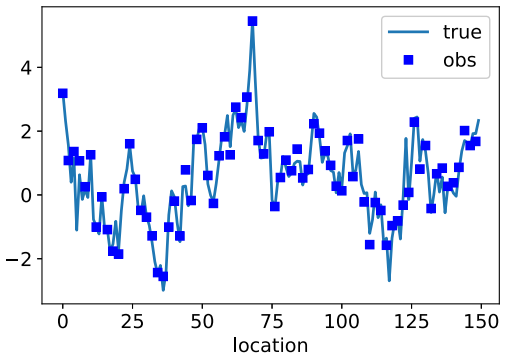

(a) The true property field and the noisy observations.

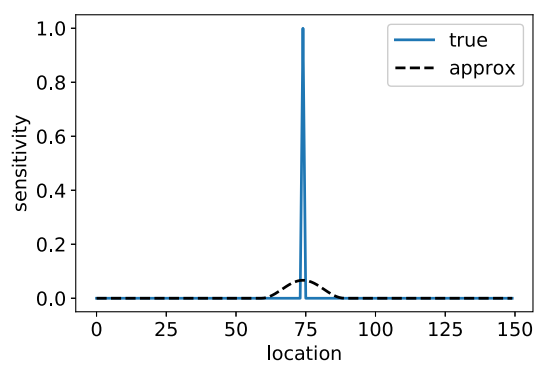

(b) True and approximate observation operators for $n=75$. observation operator that is used in the data assimilation. For a uniform field, both operators would return the same values, but they return different values when applied to heterogeneous fields.

Starting with an initial (correct) estimate of the measurement noise, the workflow of Algorithm 4 is applied to iteratively estimate the total observation error covariance. The data assimilation in each loop is performed using the ensemble Kalman smoother described in Algorithm 1 with local analysis as described in Chen and Oliver [8]. Ensemble size was 60 . The Gaspari-Cohn correlation function [25] with range $2 a=45$ was used for observation tapering. We assume that the covariance of the total observation error is stationary so that we can use the methodology of Algorithm 3 to generate new realizations of data noise by shifting current realizations. Figure 2a shows the ensemble of predicted observations $(\tilde{\mathbf{h}}(\boldsymbol{\theta}))$ after assimilation using $\mathbf{C}_{D}^{(0)}$, which was based only on measurement noise. To make a comparison with the observations, we must perturb the predicted observations (after data assimilation) with noise from the estimated distribution. The comparison in Fig. $2 b$ shows that the spread in the posteriori ensemble is far too small and does not cover the observations. After iteratively improving the estimate of the total observation error covariance, we again compute the ensemble of predicted observations (Fig. 2c) and the a posteriori ensemble of perturbed predictions (Fig. 2d). The improvement in the coverage is clear.

Although we do not require or form the estimates of $\mathbf{C}_{D}^{(i)}$ for $i>0$, it is instructive to visualize the evolution of the estimates in this test case (Fig. 2e). Because we assume stationarity of the total observation error covariance, a single row of the covariance matrix is sufficient to characterize the entire matrix. Note that at iteration 0 , the variance is 0.04 and that the value of the variance increases quickly to a value near 1.0. The covariance also quickly develops a short-range correlation. The lack of smoothness is due to the small domain and the influence of the single realization of the "true" observations.

In practice, iterative improvement would terminate when the posteriori model diagnostic (Eq. 2) attains a value that is consistent with the expected distribution of values. Figure $2 \mathrm{f}$ shows that the value of the model diagnostic (probability $p$ in Algorithms 2 and 4) is acceptable (larger than 0.05) after one iteration. This is consistent with the findings of Michel [38], who showed in a related approach that a single iteration was often sufficient to obtain a good estimate of a scale factor for the error covariance.

Because the test problem is relatively small (75 observations and 150 model parameters), it is possible to compute the iterative improvement in the estimate of the covariance for total observation error that would be obtained if it were possible to use Eq. 17 directly. The initial estimate of observation error covariance (iteration 0 ) is pure nugget with a variance of 0.04 . It is the variance of the actual measurement error for the true observation operator. After a single iteration, the estimate of observation error variance increases to nearly 1.0 and the errors become correlated (Fig. 3a). Further iterations slowly approach the theoretical total observation error covariance which has a variance of approximately 1.75 (Fig. 3b). Although convergence is slow, we note that even a single iteration results in an estimate of the covariance that is far better than the initial estimate.

The purpose of data assimilation is to make predictions. Because this is a synthetic example, however, it is possible to compare updated estimates of the property field with the true property field from which the observations were obtained. In the linear 1D test case, the observation operator used for data assimilation was intentionally made to be incorrect. If data assimilation is performed using the incorrect forward model with the actual observation error covariance, the results are an underestimation of uncertainty in the updated model parameters, and biased predictions (Fig. 4a). If the methodology described in Algorithm 4 is used, however, the spread in the final ensemble is increased and the ensemble of updated parameters appears to be consistent with the truth (Fig. 4b).

The quality of the final ensemble is quantified using a formulation of the Mahalanobis distance for high dimensions. We use cross-validation to estimate the probability that the truth was drawn from the same distribution as the ensemble of posterior realizations. Using the initial estimate of 


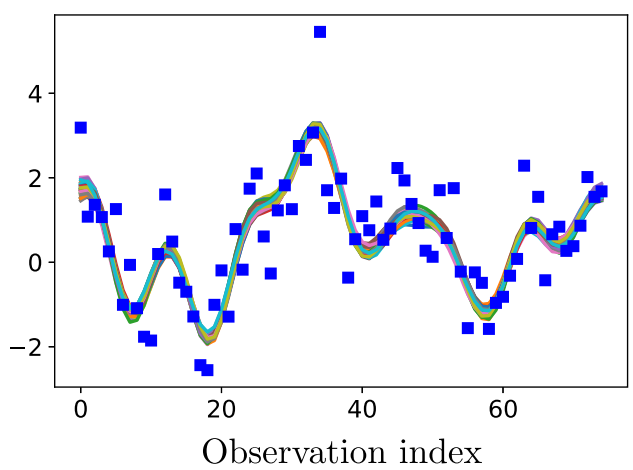

(a) Predicted observations from the updated ensemble using initial $C_{D}$

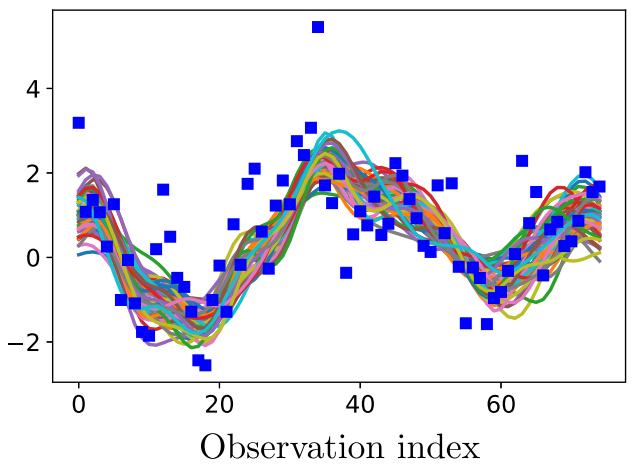

(c) Predicted observations from the updated ensemble after correcting $C_{D}$

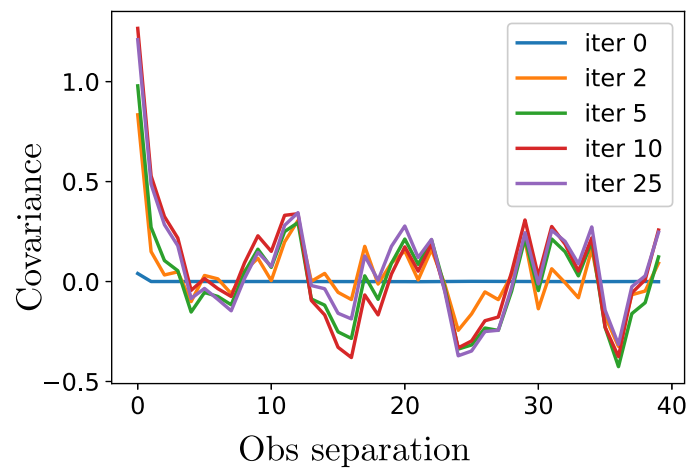

(e) Several iterations of the first row of sample estimate of $C_{D}$. Errors are correlated.

Fig. 2 Results from the linear 1D example. In this example, the number of observations is 75 and the ensemble size is 60 . Local analysis with data tapering was used for data assimilation. a Predicted observations from the updated ensemble using initial $C_{D}$. b Perturbed predicted observations from the updated ensemble using initial $C_{D}$. c Predicted observations from the updated ensemble after correcting

$\mathbf{C}_{D}$, the probability for Fig. $4 \mathrm{a}$ is less than 0.0005 , while the probability for the truth to be drawn from the same distribution as the ensemble in Fig. $4 \mathrm{~b}$ is approximately 0.49 .

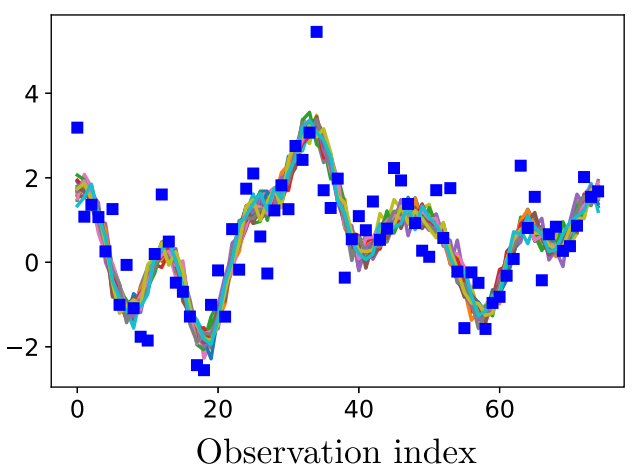

(b) Perturbed predicted observations from the updated ensemble using initial $C_{D}$

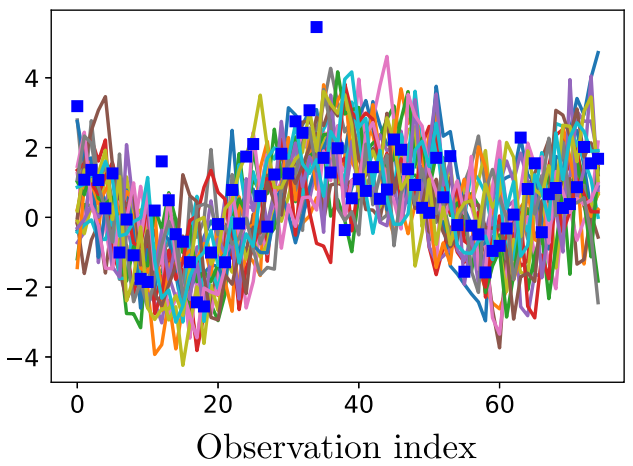

(d) Perturbed predicted observations from the updated ensemble after correcting $C_{D}$

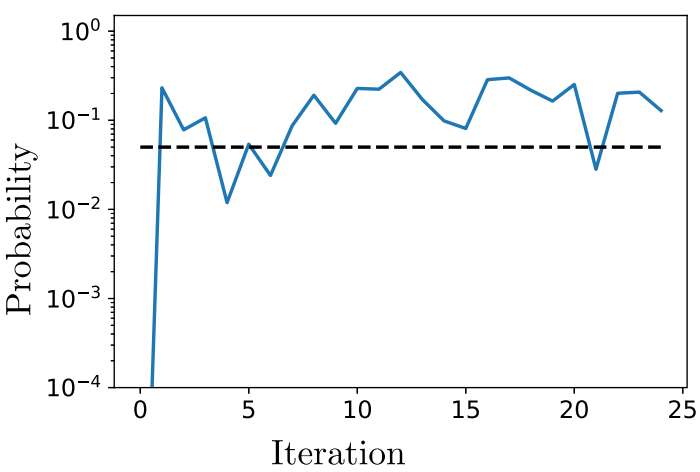

(f) The first 25 values of the posterior model diagnostic (probability $p$ in Algorithms 2 and 4) for data residuals.

$C_{D}$. d Perturbed predicted observations from the updated ensemble after correcting $C_{D}$. e Several iterations of the first row of sample estimate of $C_{D}$. Errors are correlated. $\mathbf{f}$ The first 25 values of the posterior model diagnostic (probability $p$ in Algorithms 2 and 4) for data residuals

\subsection{Norne Field example}

In this section, we apply the workflow and methodology of Algorithm 4 to a much larger problem for which the 
Fig. 3 Iterative estimation of $C_{D}$ for infinite ensemble size. a Early iterations (but the initial guess is not shown). b Later iterations and the theoretical covariance

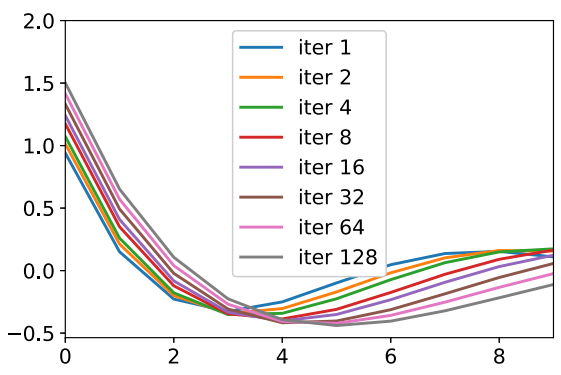

(a) Early iterations (but the initial guess is not shown).

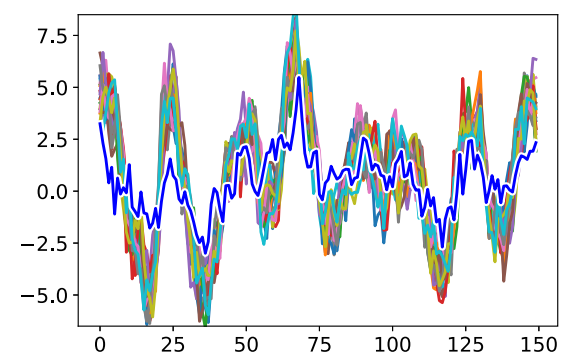

(a) Updated model parameters after data assimilation with initial estimate of $\mathbf{C}_{D}$.

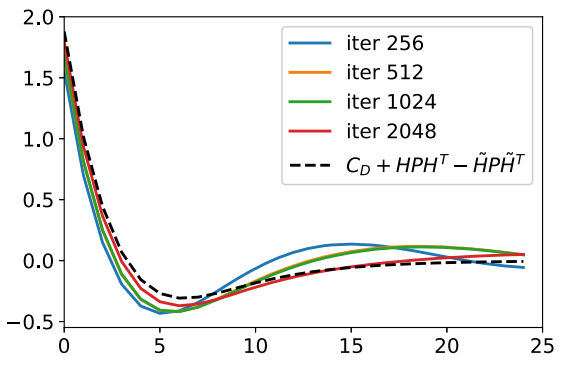

(b) Later iterations and the theoretical covariance.

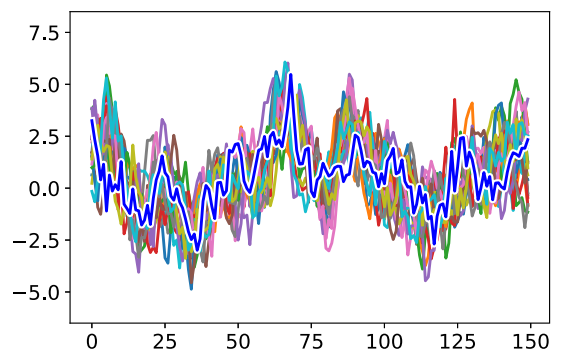

(b) Updated model parameters after data assimilation with final estimate of $\mathbf{C}_{D}$.
Fig. 5 True reservoir model property fields in layer 11 of the Norne Field model

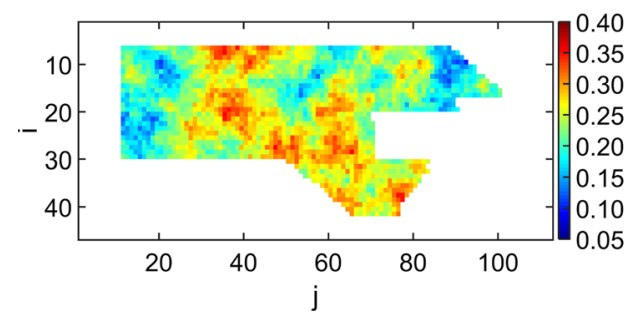

true porosity

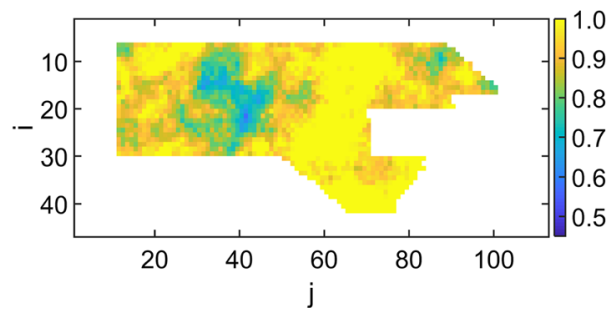

true NTG

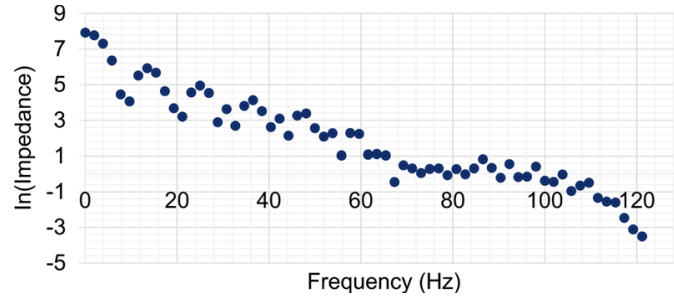

(a) Frequency spectrum computed from the Norne 2001 impedance volume.

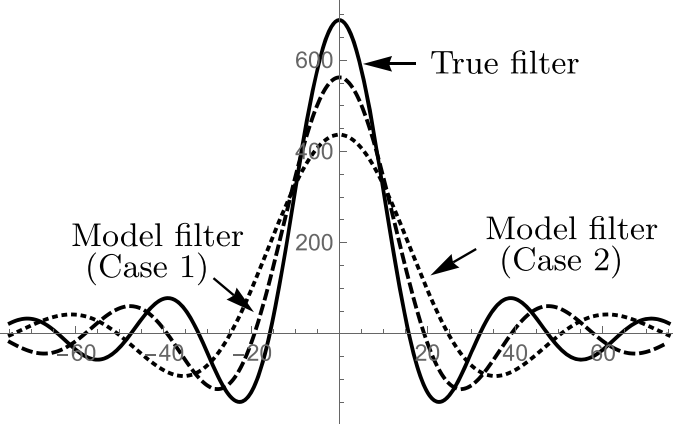

(b) True and approximate observation operators.

Fig. 6 Model error due to imperfect vertical filtering. a Frequency spectrum computed from the Norne 2001 impedance volume. b True and approximate observation operators 
Fig. 7 Two realizations of seismic noise in layer 11 generated from the noise model estimated using factorial co-kriging on the 2001 and 2006 seismic surveys. Units of impedance: $(\mathrm{m} / \mathrm{s}) .(\mathrm{g} / \mathrm{cc})$
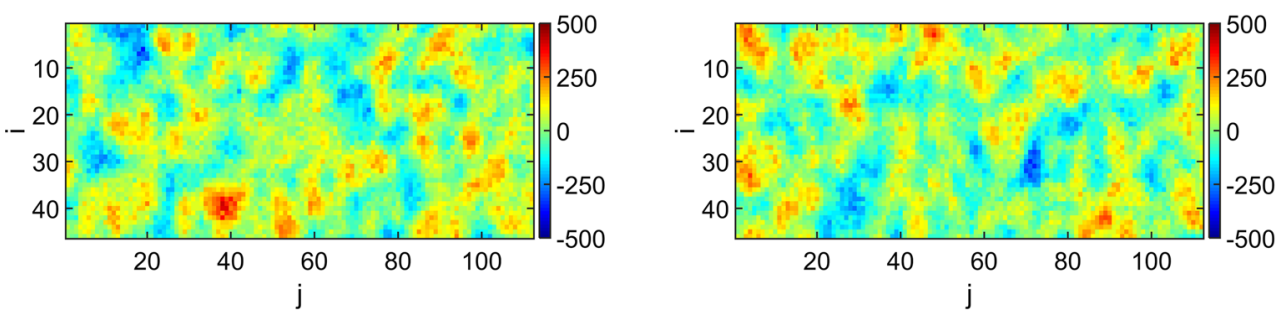

computation and use of a non-diagonal observation error covariance matrix, $\mathbf{C}_{D}$, might be problematic using standard methods. The synthetic test problem that we have chosen is based on the Norne Field benchmark case [51]. In our test problem, we assimilate synthetic acoustic impedance data to update estimates of porosity and net-to-gross ratio in each active cell of the Norne simulation model at one reservoir condition. We intentionally add model error to the problem so that an accurate estimate of observation noise is not sufficient to explain the discrepancy between the observations and the model predictions.

Our models for assimilation of seismic data are based on the Norne reservoir simulation model provided by the I/O Centre at the Norwegian University of Science and Technology (NTNU). The model is composed of 46 cells by 112 cells by 22 cells in the $i, j$, and $k$ directions respectively, with a total of 44,927 active cells. The fourth layer, representing the Not Shale Formation, consists entirely of inactive cells. The average horizontal cell dimensions in this model are approximately $100 \mathrm{ft}$ by $100 \mathrm{ft}$ (30.48 m by
$30.48 \mathrm{~m}$ ). One realization of porosity and NTG from Chen and Oliver [7] has been used to define the true porosity and true NTG fields in this work (Fig. 5). This realization is not a part of the 100 model realizations that are updated during data assimilation.

In order to simulate seismic data from a reservoir simulation model, we minimally require a rock-physics or petro-elastic model (PEM), a filtering function, a seismic noise model, and time-to-depth conversion. A PEM is a set of relationships that aim to convert certain reservoir properties (e.g., porosity and NTG) and reservoir variables (e.g., saturation, pressure) into elastic properties, such as velocities and density. Synthetic seismic acoustic impedance data are created using a "true" petro-elastic model based on the Gassmann's equations [26, 62], the Hashin-Shtrikman bound models [27] for a mixture of sands and shales, and the fluid and mineral parameters proposed for the Norne Field [12]. For the Hashin-Shtrikman bounds, we assume that the volume of shale in each of the active cells in the model is given by 1-NTG [3]. The true PEM
Fig. 8 True model impedance (top row) is computed at the simulator gridblock level. True seismic impedance (middle row) is obtained by filtering the true model impedance using the "true" filter. Observed seismic impedance (bottom row) is obtained by adding a realization of correlated seismic noise to the true seismic impedance. Only horizontal layer 11 and vertical section 11 are shown. Units of impedance: $(\mathrm{m} / \mathrm{s}) .(\mathrm{g} / \mathrm{cc})$
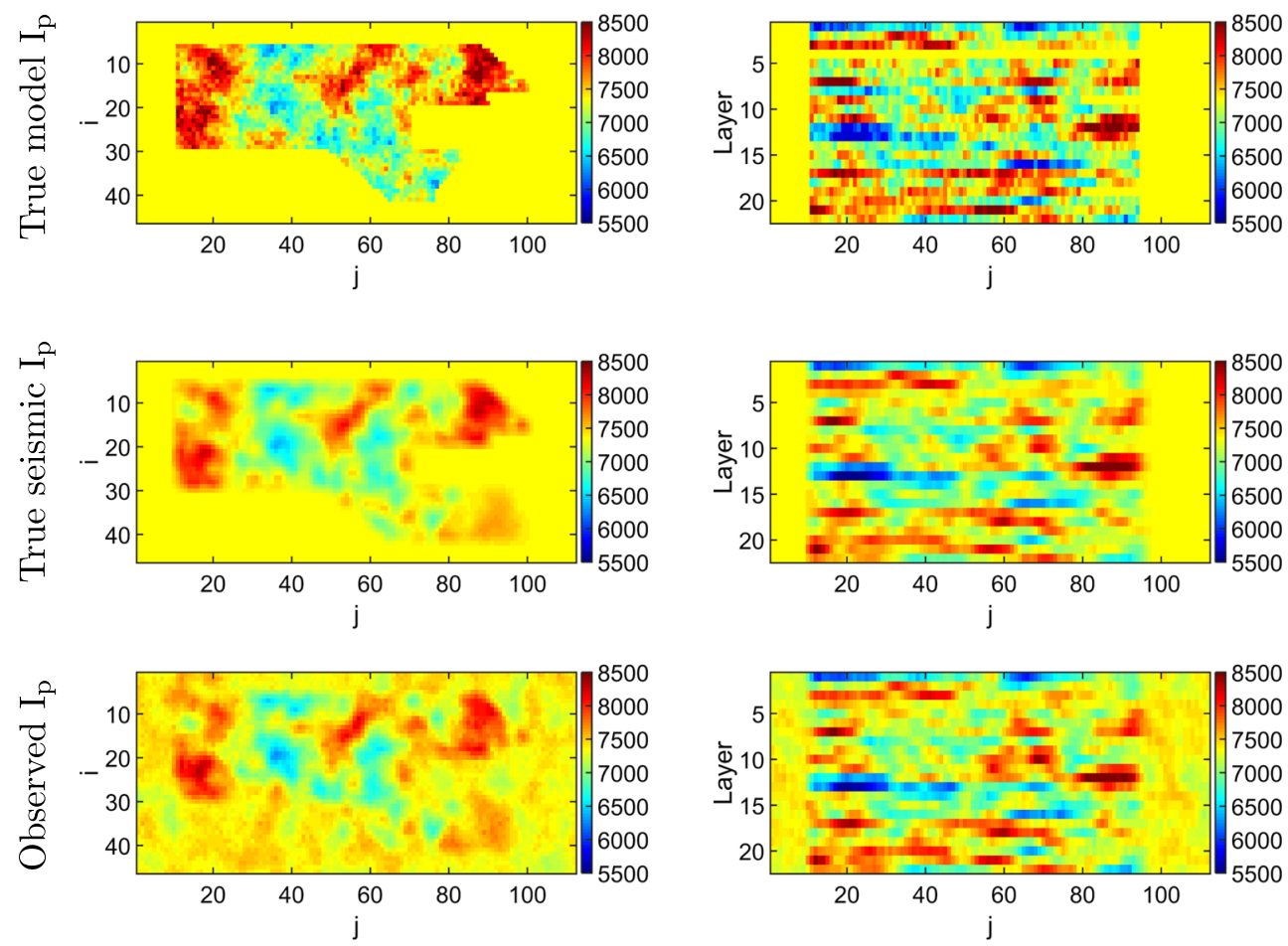

Layer 11

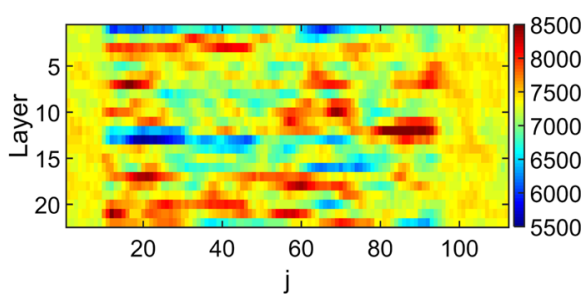

Section 11 
Table 1 Test cases used for the Norne Field example. The first case (Case 1) is characterized by a large model error coming from a biased petro-elastic model (PEM) and filtering step. In the second example (Case 2), with smaller model error, we use a biased vertical (frequency) filter

\begin{tabular}{llll}
\hline Test cases & Case 1 & Case 2 & Truth \\
\hline Petro-elastic model & $\begin{array}{l}\text { Biased dry moduli vs. } \\
\text { porosity relationships } \\
\text { Error in shale volume }\end{array}$ & True PEM & True PEM \\
$\begin{array}{l}\text { Horizontal filter } \\
\text { (moving average) }\end{array}$ & $5 \times 5$ & $3 \times 3$ & $3 \times 3$ \\
$\begin{array}{l}\text { Vertical filter } \\
\text { (Ormsby) }\end{array}$ & $0-0-80-100 \mathrm{~Hz}$ & $0-0-60-80 \mathrm{~Hz}$ & $0-0-100-120 \mathrm{~Hz}$ \\
\hline
\end{tabular}

is then used to simulate the true impedance field from this realization (Fig. 8, top row).

In this paper, seismic observations and predictions are compared at the scale of the Norne seismic impedance data. A "true" vertical frequency filter is created based on the frequency spectrum of the inverted acoustic impedance data from the 2001 Norne seismic dataset (Fig. 6a). To approximately match the frequency spectrum of the real Norne impedance data, we selected a low-pass Ormsby filter [46], with cut frequencies at 0-0-100-120 Hz. For a sandstone velocity of $3500 \mathrm{~m} / \mathrm{s}$, this corresponds approximately to the sensitivity function in depth shown by the solid curve in Fig. 6b. To model the horizontal resolution of the inverted acoustic impedance, we further apply a horizontal moving-average filter with a window of three cells by three cells. The Norne Field data set only provides a reservoir simulation model, not a geomodel. In order to apply the filtering to generate seismic predictions from the simulation model, it was necessary to assign impedance values to inactive cells in the model for which no petrophysical properties were assigned. In this study, we assigned the Not Shale acoustic impedance value of 7360 $(\mathrm{m} / \mathrm{s}) .(\mathrm{g} / \mathrm{cc})$ from the Norne dataset [12] to inactive cells. The filtered true seismic impedance is shown in the middle row of Fig. 8.

Noise is often estimated from seismic data by assuming that the noise is either spatially uncorrelated or that it is of a much different frequency than the signal. Because the Norne data set has multiple vintages of seismic data, it is possible to use factorial co-Kriging (FCK) [1, 10, 47] to separate noise from signal without making those assumptions. We used FCK to decompose the 2001 baseline and the 2006 monitor survey into three parts: A common part between the two surveys, and two spatially independent residuals from the baseline and the monitor survey. Our seismic noise model for the observations in the test case is based on the residuals extracted from the 2001 baseline survey. The covariance estimate for the seismic noise has a nugget effect with amplitude 983 (units of impedance squared) and a cubic covariance [9] with amplitude 8347 and ranges in the $i, j$, and $k$ directions of 10,8 , and 4 grid cells, respectively. Figure 7 shows two realizations of seismic noise generated from the initial data error covariance estimate. Seismic impedance observations, obtained by adding a realization
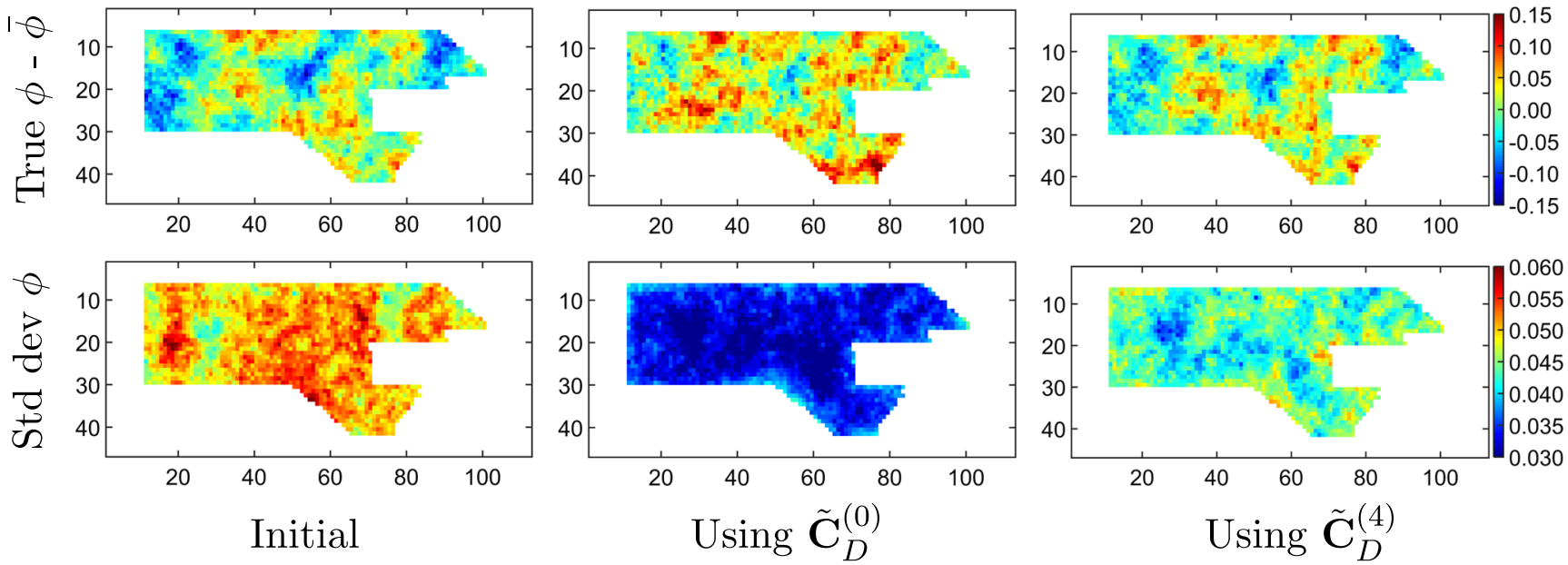

Fig. 9 Top row shows the difference between the true porosity field and the ensemble mean of the porosity field for the example with large model error (Case 1). Bottom row shows spread in the ensemble of porosity 

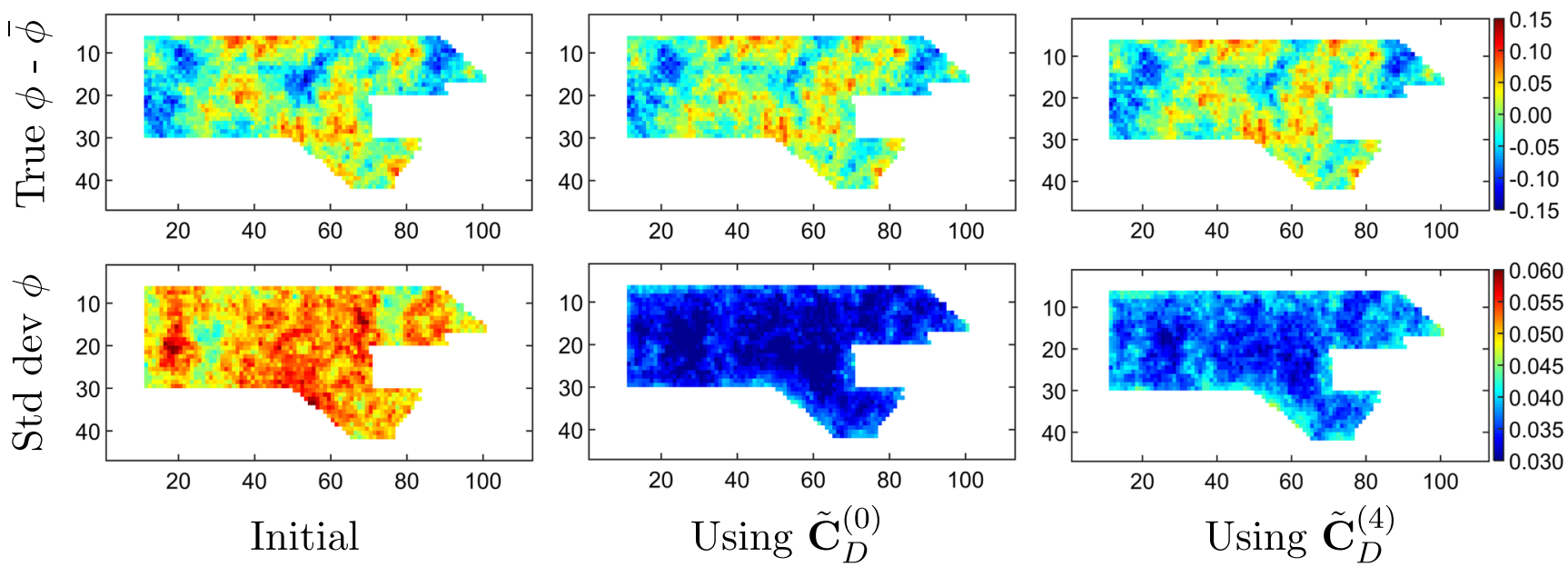

Fig. 10 Top row shows the difference between the true porosity field and the ensemble mean of the porosity field for the example with small model error (Case 2). Bottom row shows spread in the ensemble of porosity

of correlated noise to the true seismic impedance field, are shown in the bottom row of Fig. 8.

\subsubsection{Test case 1: Large model error}

In this test case, we consider two sources of model error: an incorrect parameter value in the petro-elastic model and a difference in scale between impedance observations and impedance predictions (Table 1). Firstly, we constructed a biased PEM by modifying the 'true' relationships between the dry moduli and the porosity provided in the Norne Field [12], and by adding an error on the assignment of the shale volumes in the Norne model. Secondly, we biased the true filters by reducing the cut frequencies in the vertical filtering to $0-0-80-100 \mathrm{~Hz}$, and by increasing the moving-averaging window of the horizontal filter to five cells by five cells. This resulted in impedance predictions that are smoother than the impedance observations in both the horizontal and vertical axes.

In this case of large model error (Case 1), the stopping criterion for estimation of total observation error covariance based on the magnitude of the model diagnostic $p$ was satisfied after four iterations (Fig. 11a), but we continued the model improvement cycle for one additional iteration of Algorithm 4. The sequence of model diagnostics based on the ensemble of residuals is shown by the solid curve in Fig. 11a. An increasing probability suggests that the magnitude of the data residuals are consistent with the estimated total observation error. Updated estimates of the total observation error covariance are shown in Fig. 12a. The result of the iteration is to monotonically increase the magnitude of the variance and to increase the range of the correlation, thus placing less weight on the observations in each cycle of model improvement. The initial estimate of $\mathbf{C}_{D}$ based on actual measurement error (pink curve) is much smaller than the final estimate. Subsequent changes in $\mathbf{C}_{D}$ are smaller than the first change, and changes in iterations $3-5$ are very small.

Because this is a synthetic example, it is possible to compare the true property fields with the ensemble of predictions. Our comparison is based on the probability that the truth is drawn from the same distribution as the ensemble
Fig. 11 Evaluation of model diagnostic used for the stopping criterion in Algorithm 4 (a), and comparison of the true porosity distribution with the ensemble after history matching for the Norne seismic test cases (b). Case 1 has large model error. Case 2 has smaller model error

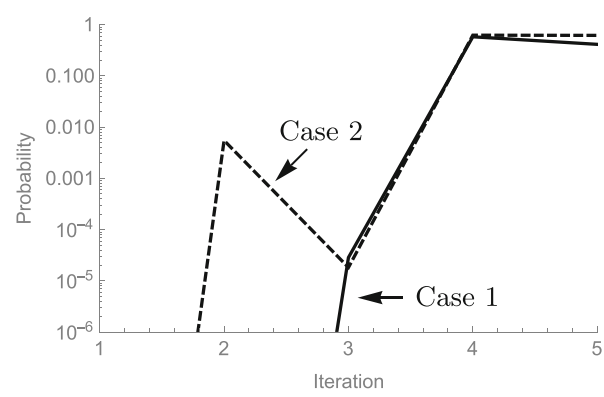

(a) The probability that the data residuals are samples from $N\left[0, \mathbf{C}_{D}\right]$.

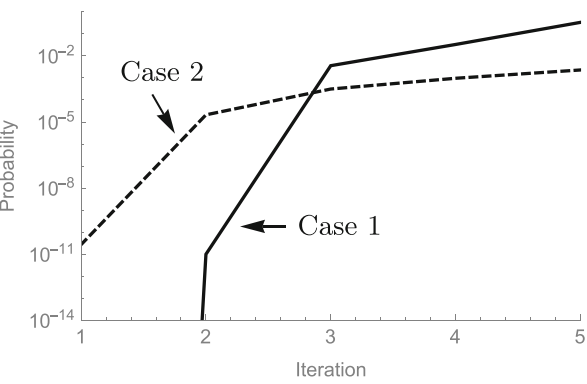

(b) The probability that the true porosity field is a sample from the posterior distribution of porosity fields. 
Fig. 12 Experimental covariance in the $j$-direction of data residuals computed using the entire ensemble. Curves show the iterative changes in the estimate of the covariance with iterations of Algorithm 4

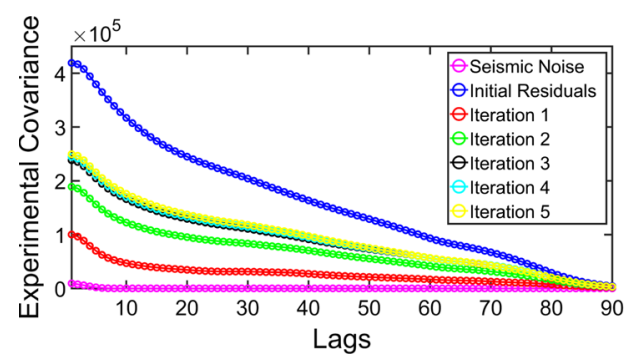

(a) Case 1, large model error.

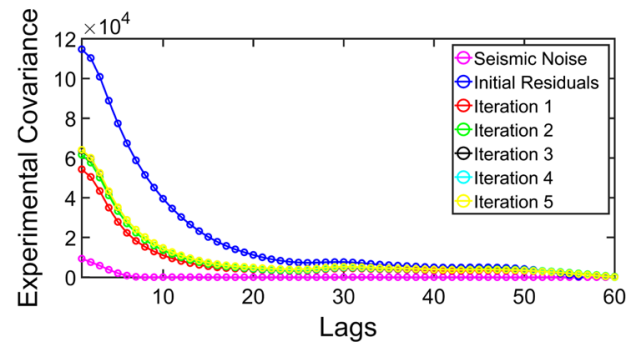

(b) Case 2, small model error. of predictions. When the actual measurement error is used in data assimilation, the truth is quite different from the predictions, but after modification of the estimate of $\mathbf{C}_{D}$ to account for model error, the difference between the truth and the ensemble of predictions becomes much smaller (Figs. 9 and $11 \mathrm{~b})$.

In summary, the model error in Case 1 was very largeso large that after data assimilation with the estimated total observation error the data were effectively ignored. We emphasize that there was nothing 'wrong' with the data in Case 1, but because the model was deficient, improvements in predictions from the model after data assimilation were small. If this situation were to occur in practice, the first recourse should be to try to improve the model by identifying missing parameters, or by increasing uncertainty, or by improving the forward simulator. After that has been done as well as possible, then the next step should be to update the estimate of $\mathbf{C}_{D}$ as was done here. The result is an ensemble estimate with smaller spread than the initial ensemble (Fig. 9), but one which is still consistent with the true porosity.

\subsubsection{Test case 2: Small model error}

In the second Norne Field example, the only source of model error is a difference in scale in the forward filtering operator (Table 1). The vertical filter used for prediction has cut frequencies of $0-0-60-80 \mathrm{~Hz}$ instead of the true cut frequencies of $0-0-100-120 \mathrm{~Hz}$. In this case, there is no error in the PEM. Similar to Case 1, the stopping criterion for estimation of total observation error covariance based on magnitude of data residuals was satisfied in Case 2 after four iterations (Fig. 11a), but we again continued the model improvement cycle for one additional iteration of Algorithm 4. The sequence of model diagnostics based on the ensemble of residuals is shown by the dashed curve in Fig. 11a.

The changes in the iterative estimates of the total observation error covariance (Fig. 12b) are not as large as in Case 1 because the magnitude of the model error is smaller. We still see, however, a change from an initial estimate of $\mathbf{C}_{D}$ that is the sum of small "nugget" effect and a cubic covariance with a range of approximately 8 grid cells (pink curve) to an estimate that is spatially correlated with a range of approximately 20 grid cells (Fig. 12b). The range of the correlated error in Case 2 is substantially smaller than the range observed in Case 1, where the sources of the model error included an error in the PEM. Changes to the estimate of $\mathbf{C}_{D}$ in iterations 2-5 are very small. The difference between true porosity and the ensemble of predictions becomes smaller after using the total estimate of $\mathbf{C}_{D}$ based on the data residuals (Fig. 10). Furthermore, the spread increases with the new total estimate of $\mathbf{C}_{D}$ as less weight is placed on the observations. The probability that the truth is drawn from the same distribution as the ensemble of calibrated predictions is smaller than expected in this case (dashed curve in Fig. 11b), possibly due to inadequate localization. The final ensemble, however, becomes closer to the truth with the iterations of Algorithm 4 (Fig. 11b).

\section{Conclusions}

All models have errors. The purpose of model calibration, model criticism and model improvement is to reduce the effects of those errors so that forecasts made from calibrated models provide reliable predictions of future behavior. If model errors are not accounted for properly, the result is overconfidence in incorrect predictions. The ultimate result is suboptimal decisions on reservoir management and development.

In this paper, we presented a procedure for identification of the presence of model error and a method for reducing the effect of model error when it cannot be removed through model improvement. The method involves iteratively updating an estimate of an effective observation error that accounts for the presence of model error.

In order to handle model error and correlated observation error in very large problems, it was necessary to develop methods that allow model checking for large models. This requires estimation of the inverse of a low-rank approximation of the observation error covariance matrix $\mathbf{C}_{D}$. We used a shrinkage-based regularization of the covariance to develop model diagnostics that can be 
used in very high dimensions. It was also necessary to perform data assimilation without forming the observation error covariance matrix. That was accomplished using a low-rank updating scheme [21], with a new method for generating unconditional realizations of sample error from a distribution for which we had only a low-rank approximation of the covariance matrix.

The methodology was tested on several synthetic problems for which the model error could be controlled and for which errors in predictions could be evaluated. In the 1D linear example, the value of the model diagnostic is acceptable after just one iteration. The second example was based on the Norne Field benchmark case. The number of observations was about 114,000 and the number of model parameters was about 90,000. Model errors were caused by errors in the petro-elastic model and by errors in the filtering function (forward operator). In both cases, the model improvement iterations (using total $\mathbf{C}_{D}$ ) increased the spread in the ensemble, which lead to less overfitting to the observations. In real applications with actual inverted seismic data, this outcome is desirable in order to avoid overfitting to biased observations. Furthermore, the resulting ensembles of porosity and NTG have a spread that is smaller than that in the initial ensemble, but that is consistent with their true fields.

We showed that the methodology described in this paper is feasible for large problems (large number of model parameters and large amounts of data), and that the application resulted in improved predictability and reduced tendency for overconfidence.

Acknowledgments We are grateful to Geovariances for providing a license for the use of Isatis for factorial co-kriging. Additionally, the authors thank Equinor (operator of the Norne Field) and its license partners Eni Norge and Petoro for the release of the Norne data. The authors acknowledge the Center for Integrated Operations at NTNU for cooperation and coordination of the Norne Cases. The view expressed in this paper are the views of the authors and do not necessarily reflect the views of Equinor and the Norne license partners.

Funding information Primary support for the authors has been provided by the CIPR/IRIS cooperative research project "4D Seismic History Matching" which is funded by industry partners Eni Norge, Petrobras, and Total, as well as the Research Council of Norway through the Petromaks2 program.
Open Access This article is distributed under the terms of the Creative Commons Attribution 4.0 International License (http:// creativecommons.org/licenses/by/4.0/), which permits unrestricted use, distribution, and reproduction in any medium, provided you give appropriate credit to the original author(s) and the source, provide a link to the Creative Commons license, and indicate if changes were made.

\section{Appendix: Expectation for residuals with incorrect forward model}

The differences between observations and predictions from models provide information about the magnitude of model error and the magnitude of observation error, but the relationship is not simple. In this appendix, we show that the expected covariance for data residuals based on the difference between predictions from a perturbed observation method of data assimilation and the actual observations takes a relatively simple form.

$r_{\text {post }}=\tilde{H} \theta^{\text {post }}-d^{\text {obs }}$

where $\tilde{H}$ is an approximation to the 'true' forward model, $H . \theta^{\text {post }}$ is the ensemble of model parameters conditioned to the observations, $d^{\text {obs }}$ is the vector of observations, and $r_{\text {post }}$ is the ensemble of data residuals.

For simplicity, we make the simplifying assumption that the "truth" can be obtained from a model that has the same parameters as our approximate model and that the model error is due to deficiency of the forward model. Define

$$
\begin{aligned}
d^{\text {obs }} & =d^{\text {true }}+\epsilon_{d} \\
& =H\left(\theta^{\text {pr }}+\epsilon_{\theta, 2}\right)+\epsilon_{d}
\end{aligned}
$$

where $\epsilon_{d} \sim N(0, R)$ and $\epsilon_{\theta, 2} \sim N(0, P)$.

Because we are assuming a gaussian prior and a linear observation operator with Gaussian errors, we use the method of randomized maximum likelihood (RML) to sample from the posterior conditional to observations [41, 45]. In this case, however, we allow for the possibility that our forward model is imperfect and that our estimate of the observation error is incorrect. A sample of the model parameters from the conditional pdf can be generated by minimizing an objective function

$\theta^{\text {post }}=\arg \min _{\theta}\left(\left(\theta-\theta^{*}\right)^{\mathrm{T}} P^{-1}\left(\theta-\theta^{*}\right)+\left(\tilde{H} \theta+\tilde{\epsilon}_{d}-d^{\mathrm{obs}}\right)^{\mathrm{T}} \tilde{R}^{-1}\left(\tilde{H} \theta+\tilde{\epsilon}_{d}-d^{\mathrm{obs}}\right)\right)$

the solution of which is

$$
\begin{aligned}
\theta^{\mathrm{post}} & =\theta^{*}-P \tilde{H}\left(\tilde{H} P \tilde{H}^{\mathrm{T}}+\tilde{R}\right)^{-1}\left(\tilde{H} \theta^{*}+\tilde{\epsilon}_{d}-d^{\mathrm{obs}}\right) \\
& =\theta^{*}-\tilde{K}\left(\tilde{H} \theta^{*}+\tilde{\epsilon}_{d}-d^{\mathrm{obs}}\right)
\end{aligned}
$$

where we defined the gain matrix based on the approximate forward model

$$
\tilde{K}=P \tilde{H}^{\mathrm{T}}\left(\tilde{H} P \tilde{H}^{\mathrm{T}}+\tilde{R}\right)^{-1} .
$$




\section{A.1 The difference between predictions from calibrated models and observations}

The difference between the calibration of perturbed observations (RML) and the actual observations is

$$
\begin{aligned}
r & =\tilde{H} \theta^{\text {post }}-d^{\mathrm{obs}} \\
& =\tilde{H} \theta^{*}-d^{\mathrm{obs}}-\tilde{K}\left(\tilde{H} \theta^{*}+\tilde{\epsilon}_{d}-d^{\mathrm{obs}}\right) \\
& =(I-\tilde{H} \tilde{K})\left(\tilde{H} \theta^{*}+\tilde{\epsilon}_{d}-d^{\mathrm{obs}}\right)-\tilde{\epsilon}_{d} .
\end{aligned}
$$

The covariance of these residuals is approximately

$E\left[r r^{\mathrm{T}}\right]=(I-\tilde{H} \tilde{K})\left(\left(H P H^{\mathrm{T}}+R\right)-\left(\tilde{H} P \tilde{H}^{\mathrm{T}}+\tilde{R}\right)\right)(I-\tilde{H} \tilde{K})^{\mathrm{T}}+\tilde{R}$

where we assume that $(\tilde{H}-H) \theta^{\mathrm{pr}}=0$. Note that if $\tilde{H}=H$ and $\tilde{R}=R$, then Eq. 14 simplifies to

$E\left[r r^{\mathrm{T}}\right]=R$

which is what we expect.

Also, note that if $\tilde{H} P \tilde{H}^{\mathrm{T}}+\tilde{R}=H P H^{\mathrm{T}}+R$, then $E\left[r r^{\mathrm{T}}\right]=\tilde{R}$. For this case, we have

$\tilde{R}=R+H P H^{\mathrm{T}}-\tilde{H} P \tilde{H}^{\mathrm{T}}$

\section{A.2 Iteration}

We use an iterative fixed-point scheme for estimation of the total observation covariance based on the residuals as shown in Eq. 14.

$\tilde{R}_{\ell+1}=\left(I-\tilde{H} \tilde{K}_{\ell}\right)\left(\left(H P H^{\mathrm{T}}+R\right)-\left(\tilde{H} P \tilde{H}^{\mathrm{T}}+\tilde{R}_{\ell}\right)\right)\left(I-\tilde{H} \tilde{K}_{\ell}\right)^{\mathrm{T}}+\tilde{R}_{\ell}$

The iterative scheme has a stable fixed-point solution when

$R+H P H^{\mathrm{T}}-\tilde{H} P \tilde{H}^{\mathrm{T}}$

is positive definite. See the discussion of convergence on page 262 of Ménard [37].

\section{References}

1. Abreu, C.E., Lucet, N., Nivlet, P., Royer, J.-J.: Improving 4D seismic data interpretation using geostatistical filtering. In: 9th International Congress of the Brazilian Geophysical Society (2005)

2. Amini, H.: A pragmatic approach to simulator-to-seismic modelling for 4D seismic interpretation. Ph.D. thesis, Heriot-Watt University (2014)

3. Amini, H., Alvarez, E., MacBeth, C., Shams, A.: Finding a petro-elastic model suitable for sim2seis calculation. In: 74th EAGE Conference and Exhibition incorporating (EUROPEC 2012) (2012)

4. Blouin, M., Le Ravalec, M., Gloaguen, E., Adelinet, M. Porosity estimation in the fort worth basin constrained by $3 \mathrm{D}$ seismic attributes integrated in a sequential Bayesian simulation framework. Geophysics 82(4), M67-M80 (2017)
5. Brynjarsdóttir, J., O’Hagan, A.: Learning about physical parameters: the importance of model discrepancy. Inverse Probl. 30(11), 114007 (2014)

6. Chen, Y., Oliver, D.S.: Levenberg-Marquardt forms of the iterative ensemble smoother for efficient history matching and uncertainty quantification. Comput. Geosci. 17(4), 689-703 (2013)

7. Chen, Y., Oliver, D.S.: History matching of the Norne full-field model with an iterative ensemble smoother. SPE Reserv. Eval. Eng. 17(2), 244-256 (2014)

8. Chen, Y., Oliver, D.S.: Localization and regularization for iterative ensemble smoothers. Comput. Geosci. 21(1), 13-30 (2017)

9. Chilès, J.-P., Delfiner, P. Geostatistics: Modeling Spatial Uncertainty, 2nd edn. Wiley, New York (2012)

10. Coléou, T., Hoeber, H., Lecerf, D., et al.: Multivariate geostatistical filtering of time-lapse seismic data for an improved 4D signature. In: 73rd Ann. Intern Mtg., SEG, Expanded Abstracts (2002)

11. Courtier, P., Andersson, E., Heckley, W., Vasiljevic, D., Hamrud, M., Hollingsworth, A., Rabier, F., Fisher, M., Pailleux, J.: The ECMWF implementation of three-dimensional variational assimilation (3D-Var). I: Formulation. Q. J. Roy. Meteorol. Soc. 124(550), 1783-1807 (1998)

12. Dadashpour, M.: Reservoir characterization using production data and time-lapse seismic data. Ph.D. dissertation NTNU, Trondheim, Norway (2009)

13. Del Giudice, D., Honti, M., Scheidegger, A., Albert, C., Reichert, P., Rieckermann, J.: Improving uncertainty estimation in urban hydrological modeling by statistically describing bias. Hydrol. Earth Syst. Sci. 17(10), 4209-4225 (2013)

14. Dietrich, C.R., Newsam, G.N.: Fast and exact simulation of stationary Gaussian processes through circulant embedding of the covariance matrix. SIAM J. Sci. Comput. 18(4), 1088-1107 (1997)

15. Doherty, J., Welter, D.: A short exploration of structural noise. Water Resour. Res. 46(5), W05525 (2010)

16. Doyen, P.M., Psaila, D.E., den Boer, L.D., Jans, D.: Reconciling data at seismic and well $\log$ scales in 3 -D earth modelling. In: Proc. of the SPE Annual Technical Conference and Exhibition, pp. 5-8, San Antonio (1997)

17. Emerick, A.A.: Analysis of the performance of ensemble-based assimilation of production and seismic data. J. Pet. Sci. Eng. 139, 219-239 (2016)

18. Emerick, A.A., Reynolds, A.C.: Ensemble smoother with multiple data assimilation. Comput. Geosci. 55, 3-15 (2013)

19. Engel, J., Buydens, L., Blanchet, L.: An overview of largedimensional covariance and precision matrix estimators with applications in chemometrics. J. Chemometr. 31(4), e2880 (2007)

20. Evensen, G.: Data Assimilation: The Ensemble Kalman Filter, 2nd edn. Springer (2009)

21. Evensen, G.: The ensemble Kalman filter for combined state and parameter estimation Monte Carlo techniques for data assimilation in large systems. IEEE Control Syst Mag 29(3), 83-104 (2009)

22. Evensen, G., Eikrem, K.S.: Conditioning reservoir models on rate data using ensemble smoothers. Comput. Geosci. 22(5), 12511270 (2018)

23. Evin, G., Thyer, M., Kavetski, D., McInerney, D., Kuczera, G.: Comparison of joint versus postprocessor approaches for hydrological uncertainty estimation accounting for error autocorrelation and heteroscedasticity. Water Resour. Res. 50(3), 2350-2375 (2014)

24. Francis, A.M.: Understanding stochastic inversion: Part 2. First Break 24(12), 79-84 (2006)

25. Gaspari, G., Cohn, S.E.: Construction of correlation functions in two and three dimensions. Q. J. R. Meteorol. Soc. 125(554), 723-757 (1999) 
26. Gassmann, F.: Elastic waves through a packing of spheres. Geophysics 16, 673-685 (1951)

27. Hashin, Z., Shtrikman, S.: A variational approach to the theory of the elastic behaviour of multiphase materials. J. Mech. Phys. Solids 11(2), 127-140 (1963)

28. Hodyss, D., Nichols, N.: The error of representation: basic understanding. Tellus Series A-Dynamic Meteorology and Oceanography 67, 24822 (2015)

29. Howes, K.E., Fowler, A.M., Lawless, A.S.: Accounting for model error in strong-constraint 4D-Var data assimilation. Q. J. Roy. Meteorol. Soc. 143(704), 1227-1240 (2017)

30. Iglewicz, B., Hoaglin, D.C.: How to Detect and Handle Outliers, vol. 16. ASQ Press (1993)

31. Janjić, T., Bormann, N., Bocquet, M., Carton, J.A., Cohn, S.E., Dance, S.L., Losa, S.N., Nichols, N.K., Potthast, R., Waller, J.A., Weston, P.: On the representation error in data assimilation. Q. J. Roy. Meteorol. Soc. 144(713), 1257-1278 (2018)

32. Kalla, S., White, C.D., Gunning, J., Glinsky, M.: Downscaling multiple seismic inversion constraints to fine-scale flow models. SPE J. 14(4), 746-758 (2009)

33. Kroese, D.P., Botev, Z.I.: Spatial process simulation. In: Schmidt, V., Geometry, S.tochastic. (eds.) Stochastic Geometry, Spatial Statistics and Random Fields: Models and Algorithms, pp. 369404. Springer International Publishing (2015)

34. Ledoit, O., Wolf, M.: A well-conditioned estimator for largedimensional covariance matrices. J. Multivariate Anal. 88(2), 365-411 (Jan 2004)

35. Leung, P.L., Chan, W.Y.: Estimation of the scale matrix and its eigenvalues in the Wishart and the multivariate $\mathrm{F}$ distributions. Ann. Inst. Stat. Math. 50(3), 523-530 (1998)

36. Liu, M., Grana, D.: Stochastic nonlinear inversion of seismic data for the estimation of petroelastic properties using the ensemble smoother and data reparameterization. Geophysics 83(3), M25M39 (2018)

37. Ménard, R.: Error covariance estimation methods based on analysis residuals: theoretical foundation and convergence properties derived from simplified observation networks. Q. J. Roy. Meteorol. Soc. 142(694), 257-273 (2016)

38. Michel, Y.: Diagnostics on the cost-function in variational assimilations for meteorological models. Nonlinear Processes Geophys. 21(1), 187-199 (2014)

39. Miller, J.W., Dunson, D.B.: Robust Bayesian inference via coarsening. arXiv:1506.06101 (2015)

40. Mirouze, I., Weaver, A.T.: Representation of correlation functions in variational assimilation using an implicit diffusion operator. Q. J. Roy. Meteorol. Soc. 136(651, B), 1421-1443 (2010)

41. Oliver, D.S.: On conditional simulation to inaccurate data. Math. Geol. 28(6), 811-817 (1996)

42. Oliver, D.S.: Calculation of the inverse of the covariance. Math. Geol. 30(7), 911-933 (1998)

43. Oliver, D.S., Alfonzo, M.: Calibration of imperfect models to biased observations. Comput. Geosci. 22(1), 145-161 (2018)

44. Oliver, D.S., Chen, Y.: Recent progress on reservoir history matching: A review. Comput. Geosci. 15(1), 185-221 (2011)

45. Oliver, D.S., Reynolds, A.C., Liu, N.: Inverse Theory for Petroleum Reservoir Characterization and History Matching. Cambridge University Press, Cambridge (2008)

46. Ormsby, J.F.A.: Design of numerical filters with applications to missile data processing. J. ACM 8(3), 440-466 (1961)

47. Pardo-Igúzquiza, E., Dowd, P.A.: FACTOR2D: A computer program for factorial cokriging. Comput. Geosci. 28(8), 857-875 (2002)
48. Philip, N., Dyce, M., Whitcombe, D.: 4D amplitude significancea technique for suppressing noise in $4 \mathrm{D}$ seismic surveys. In: 71st EAGE Conference and Exhibition (2009)

49. Rainwater, S., Bishop, C.H., Campbell, W.F.: The benefits of correlated observation errors for small scales. Q. J. Roy. Meteorol. Soc. 141(693), 3439-3445 (2015)

50. Roggero, F., Lerat, O., Ding, D.Y., Berthet, P., Bordenave, C., Lefeuvre, F., Perfetti, P.: History matching of production and 4D seismic data: Application to the Girassol Field, Offshore Angola. Oil Gas Sci. Technol. - . Rev. IFP Energies nouvelles 67(2), 237-262 (2012)

51. Rwechungura, R.W., Suwartadi, E., Dadashpour, M., Kleppe, J., Foss, B.A.: The Norne Field case - a unique comparative case study. In: SPE Intelligent Energy Conference and Exhibition. Society of Petroleum Engineers (2010)

52. Satterfield, E., Hodyss, D., Kuhl, D.D., Bishop, C.H.: Investigating the use of ensemble variance to predict observation error of representation. Mon. Weather. Rev. 145(2), 653-667 (2017)

53. Schäfer, J., Strimmer, K.: A shrinkage approach to large-scale covariance matrix estimation and implications for functional genomics. Stat. Appl. Genet. Mol. Biol. 4(1, 32), 1-30 (2005)

54. Seaman, R.S.: Absolute and differential accuracy of analyses achievable with specified observational network characteristics. Mon. Weather. Rev. 105(10), 1211-1222 (1977)

55. Sengupta, M., Mavko, G., Mukerji, T.: Quantifying subresolution saturation scales from time-lapse seismic data: A reservoir monitoring case study. Geophysics 68(3), 803-814 (2003)

56. Stephen, K.D., Shams, A., MacBeth, C.: Faster seismic history matching in a United Kingdom continental shelf reservoir. SPE Reserv. Eval. Eng. 12(4), 586-594 (2007). 2007 SPE Europec/EAGE Annual Conference and Exhibition, London, England, June 11-14, 2007

57. Stewart, L.M., Dance, S.L., Nichols, N.K.: Correlated observation errors in data assimilation. Int. J. Numer. Methods Fluids 56(8), 1521-1527 (2008)

58. Thore, P.: Uncertainty in seismic inversion: What really matters? Lead. Edge 34(9), 1000-1004 (2015)

59. Trefethen, L.N., Bau, D. III.: Numerical Linear Algebra, vol. 50. SIAM (1997)

60. Waller, J.A., Ballard, S.P., Dance, S.L., Kelly, G., Nichols, N.K., Simonin, D.: Diagnosing horizontal and inter-channel observation error correlations for SEVIRI observations using observation-minus-background and observation-minus-analysis statistics. Remote Sens. 8, 7 (2016)

61. Waller, J.A., Dance, S.L., Nichols, N.K.: Theoretical insight into diagnosing observation error correlations using observationminus-background and observation-minus-analysis statistics. Q. J. Roy. Meteorol. Soc. 142(694), 418-431 (2016)

62. Wang, Z.Z.: Y2K tutorial: Fundamentals of seismic rock physics. Geophysics 66(2), 398-412 (2001)

63. Watson, J., Holmes, C.: Approximate models and robust decisions. Statist. Sci. 31(4), 465-489 (2016)

64. White, J.T., Doherty, J.E., Hughes, J.D.: Quantifying the predictive consequences of model error with linear subspace analysis. Water Resour. Res. 50(2), 1152-1173 (2014)

Publisher's note Springer Nature remains neutral with regard to jurisdictional claims in published maps and institutional affiliations. 